\title{
Critical spectrum of fluctuations for deconfinement at proto-neutron star cores
}

\author{
G. Lugones ${ }^{1}$ and A. G. Grunfeld ${ }^{2,3,4}$ \\ 1 Universidade Federal do ABC, Centro de Ciências Naturais e Humanas, \\ Rua Santa Adélia, 166, 09210-170, Santo André, Brazil \\ 2 Department of Physics, Sultan Qaboos University, \\ P.O.Box: 36 Al-Khode 123 Muscat, Sultanate of Oman \\ 3 CONICET, Rivadavia 1917, (1033) Buenos Aires, Argentina. \\ 4 Departamento de Física, Comisión Nacional de Energía Atómica, (1429) Buenos Aires, Argentina.
}

\begin{abstract}
We study the deconfinement of hadronic matter into quark matter in a protoneutron star focusing on the effects of the finite size on the formation of just-deconfined color superconducting quark droplets embedded in the hadronic environment. The hadronic phase is modeled by the non-linear Walecka model at finite temperature including the baryon octet and neutrino trapping. For quark matter we use an $S U(3)_{f}$ Nambu-Jona-Lasinio model including color superconductivity. The finite size effects on the just deconfined droplets are considered in the frame of the multiple reflection expansion. In addition, we consider that just deconfined quark matter is transitorily out of equilibrium respect to weak interaction, and we impose color neutrality and flavor conservation during the transition. We calculate self-consistently the surface tension and curvature energy density of the quark hadron inter-phase and find that it is larger than the values typically assumed in the literature. The transition density is calculated for drops of different sizes, and at different temperatures and neutrino trapping conditions. Then, we show that energy-density fluctuations are much more relevant for deconfinement than temperature and neutrino density fluctuations. We calculate the critical size spectrum of energy-density fluctuations that allows deconfinement as well as the nucleation rate of each critical bubble. We find that drops with any radii smaller than $800 \mathrm{fm}$ can be formed at a huge rate when matter achieves the bulk transition limit of 5-6 times the nuclear saturation density.

PACS numbers: 12.39.Fe, 25.75.Nq, 26.60.Kp
\end{abstract}

\section{INTRODUCTION}

The cores of massive neutron stars have densities that may favor the nucleation of small droplets of quark matter, that under appropriate conditions may grow converting a large part of the star into quark matter (for recent work see [1-4] and references therein). Since the the equation of state above the nuclear saturation density is still uncertain the problem is usually analyzed within a two-phase description in which a hadronic model valid around the nuclear saturation density $\rho_{0}$ is extrapolated to larger densities and a quark model that is expected to be valid only for asymptotically large densities is extrapolated downwards. Within this kind of analysis some work has been performed recently in order to determine the effect of different hadronic and quark equations of state, as well as the effect of temperature, neutrino trapping and color superconductivity [1].

An important characteristic of the deconfinement transition in neutron stars, is that quark and lepton flavors must be conserved during the process leaving just deconfined quark matter that is transitorily out of equilibrium with respect to weak interactions (see [1] and references therein). When color superconductivity is included in the analysis together with flavor conservation, it is found that the most likely configuration of the just deconfined phase is two-flavor color superconductor (2SC) provided the pairing gap is large enough [5].

Since our previous studies were made in bulk, we shall now include finite size effects in the description of the just deconfined drops. To this end we employ the NambuJona-Lasinio (NJL) model for quark matter and include finite size effects within the multiple reflection expansion (MRE) framework (see [6 8 ] and references therein). Through this analysis we can determine the density of hadronic matter at which deconfinement is possible for different radii of the just formed quark drops using typical conditions expected in the interior of protoneutron stars (PNSs), i.e. temperatures in the range of $0-60 \mathrm{MeV}$ and chemical potentials of the trapped neutrino gas up to $200 \mathrm{MeV}$. Notice that to form a finite size drop it is needed some over-density with respect to the bulk transition density in order to compensate the surface and curvature energy cost. Since this energy cost depends on the drop radius $R$, so does the necessary over-density necessary to nucleate it. Thus, we can derive a critical fluctuation spectrum $(\delta \rho / \rho$ versus $R)$ delimiting which over-densities can deconfine and grow unlimitedly and which ones will shrink back to hadronic matter.

The paper is organized as follows. In Section II we briefly describe main features of the hadronic model. In Sect. III we present the quark model we use taking into account finite size effects and derive self-consistently the surface tension and the curvature energy from the MRE thermodynamic potential. In Sect IV we the find the density at which color superconducting droplets of different radii can be nucleated in protoneutron star matter. In Section $\mathrm{V}$ we find the critical spectrum of density fluctuations as well as the nucleation rate of the critical size droplets. In Sect. V we present our conclusions. 


\section{THE HADRONIC PHASE}

The hadronic phase is modeled by the non-linear Walecka model (NLWM) 9 12 including the whole baryon octet. The Lagrangian of the model is given by

$$
\mathcal{L}=\mathcal{L}_{B}+\mathcal{L}_{M}+\mathcal{L}_{L},
$$

where the indices $B, M$ and $L$ refer to baryons, mesons and leptons respectively. For the baryons we have

$$
\begin{array}{r}
\mathcal{L}_{B}=\sum_{B} \bar{\psi}_{B}\left[\gamma^{\mu}\left(i \partial_{\mu}-g_{\omega B} \omega_{\mu}-g_{\rho B} \vec{\tau} \cdot \vec{\rho}_{\mu}\right)\right. \\
\left.-\left(m_{B}-g_{\sigma B} \sigma\right)\right] \psi_{B},
\end{array}
$$

with $B$ extending over the nucleons $N=n, p$ and the following hyperons $H=\Lambda, \Sigma^{+}, \Sigma^{0}, \Sigma^{-}, \Xi^{-}$, and $\Xi^{0}$. The contribution of the mesons $\sigma, \omega$ and $\rho$ is given by

$$
\begin{aligned}
\mathcal{L}_{M}= & \frac{1}{2}\left(\partial_{\mu} \sigma \partial^{\mu} \sigma-m_{\sigma}^{2} \sigma^{2}\right)-\frac{b}{3} m_{N}\left(g_{\sigma} \sigma\right)^{3}-\frac{c}{4}\left(g_{\sigma} \sigma\right)^{4} \\
& -\frac{1}{4} \omega_{\mu \nu} \omega^{\mu \nu}+\frac{1}{2} m_{\omega}^{2} \omega_{\mu} \omega^{\mu} \\
& -\frac{1}{4} \vec{\rho}_{\mu \nu} \cdot \vec{\rho}^{\mu \nu}+\frac{1}{2} m_{\rho}^{2} \vec{\rho}_{\mu} \cdot \vec{\rho}^{\mu}
\end{aligned}
$$

where the coupling constants are

$$
g_{\sigma B}=x_{\sigma B} g_{\sigma}, \quad g_{\omega B}=x_{\omega B} g_{\omega}, \quad g_{\rho B}=x_{\rho B} g_{\rho}
$$

with $x_{\sigma B}, x_{\omega B}$ and $x_{\rho B}$ equal to 1 for the nucleons and 0.9 for hyperons. Electrons and neutrinos are included as a free Fermi gas in chemical equilibrium with all other particles.

There are five constants in the model that are determined by the properties of nuclear matter, three that determine the nucleon couplings to the scalar, vector and vector-isovector mesons $g_{\sigma} / m_{\sigma}, g_{\omega} / m_{\omega}, g_{\rho} / m_{\rho}$, and two that determine the scalar self interactions $b$ and $c$. It is assumed that all hyperons in the octet have the same coupling than the $\Lambda$. These couplings are expressed as a ratio to the nucleon couplings mentioned above, that we thus simply denote $x_{\sigma}, x_{\omega}$ and $x_{\rho}$.

In the present work we use the following values for the constants: $\left(g_{\sigma} / m_{\sigma}\right)^{2}=11.79 \mathrm{fm}^{2},\left(g_{\omega} / m_{\omega}\right)^{2}=$ $7.149 \mathrm{fm}^{2},\left(g_{\rho} / m_{\rho}\right)^{2}=4.411 \mathrm{fm}^{2}, \quad b=0.002947$ and $c=-0.001070$. This model has been labelled as GM4 in previous work [1]. The equation of state is rather stiff and gives a maximum mass of $2 \mathrm{M}_{\odot}$, that seems to be more adequate in light of the recently determined mass of the pulsar PSR J1614-2230 with $M=1.97 \pm 0.04 M_{\odot}$ [13]. This is the largest mass reported ever for a pulsar with a high precision. For more details on the explicit form of the equation of state the reader is referred to Ref. 1].

\section{THE QUARK MATTER PHASE}

The just deconfined quark matter phase is implemented by using the $S U(3)_{f}$ NJL effective model with the inclusion quark-quark interactions, which are the responsible for color superconductivity.

The corresponding Lagrangian is given by

$$
\begin{aligned}
\mathcal{L}= & \bar{\psi}(i \not \partial-\hat{m}) \psi \\
& +G \sum_{a=0}^{8}\left[\left(\bar{\psi} \tau_{a} \psi\right)^{2}+\left(\bar{\psi} i \gamma_{5} \tau_{a} \psi\right)^{2}\right] \\
& +2 H \sum_{A, A^{\prime}=2,5,7}\left[\left(\bar{\psi} i \gamma_{5} \tau_{A} \lambda_{A^{\prime}} \psi_{C}\right)\left(\bar{\psi}_{C} i \gamma_{5} \tau_{A} \lambda_{A^{\prime}} \psi\right)\right]
\end{aligned}
$$

where $\hat{m}=\operatorname{diag}\left(m_{u}, m_{d}, m_{s}\right)$ is the current mass matrix in flavor space. In the present paper we work in the isospin symmetric limit $m_{u}=m_{d}=m$. Moreover, $\tau_{i}$ and $\lambda_{i}$ with $i=1, . ., 8$ are the Gell-Mann matrices corresponding to the flavor and color groups respectively, and $\tau_{0}=\sqrt{2 / 3} 1_{f}$. Finally, the charge conjugate spinors are defined as follows: $\psi_{C}=C \bar{\psi}^{T}$ and $\bar{\psi}_{C}=\psi^{T} C$, where $\bar{\psi}=\psi^{\dagger} \gamma^{0}$ is the Dirac conjugate spinor and $C=i \gamma^{2} \gamma^{0}$.

In order to determine the relevant thermodynamic quantities we have to obtain the grand canonical thermodynamic potential at finite temperature $T$ and chemical potentials $\mu_{f c}$. Here, $f=(u, d, s)$ and $c=(r, g, b)$ denotes flavor and color indices respectively. In the following we present the thermodynamic potential for the bulk system and then, in Sect. III B, we discuss the effects of finite size in the effective potential, for the spherical droplets.

\section{A. Quark matter in bulk}

Starting from Eq. (5), we perform the usual bosonization of the theory. We introduce the scalar and pseudoscalar meson fields $\sigma_{a}$ and $\pi_{a}$ respectively, together with the bosonic diquark field $\Delta_{A}$. In what follows we will work within the mean field approximation (MFA), in which these bosonic fields are expanded around their vacuum expectation values and the corresponding fluctuations neglected. For the meson fields this implies $\hat{\sigma}=\sigma_{a} \tau_{a}=\operatorname{diag}\left(\sigma_{u}, \sigma_{d}, \sigma_{s}\right)$ and $\pi_{a}=0$. Concerning the diquark mean field, we will assume that in the density region of interest only the $2 \mathrm{SC}$ phase might be relevant. Thus, we adopt the ansatz $\Delta_{5}=\Delta_{7}=0$, $\Delta_{2}=\Delta$. Integrating out the quark fields and working in the framework of the Matsubara and Nambu-Gorkov formalism we obtain the following MFA quark thermodynamic potential $\Omega_{q}^{M F A}\left(T, \mu_{f c}, \sigma_{u}, \sigma_{d}, \sigma_{s},|\Delta|\right)$ per unit volume (further calculation details can be found in Refs. [14 17])

$$
\begin{aligned}
\frac{\Omega_{q}^{M F A}}{V}= & 2 \int_{0}^{\Lambda} \frac{k^{2} d k}{2 \pi^{2}} \sum_{i=1}^{9} \omega\left(x_{i}, y_{i}\right)+ \\
& \frac{1}{4 G}\left(\sigma_{u}^{2}+\sigma_{d}^{2}+\sigma_{s}^{2}\right)+\frac{|\Delta|^{2}}{2 H},
\end{aligned}
$$


where $\Lambda$ is the cut-off of the model and $\omega(x, y)$ is defined by

$$
\begin{array}{r}
\omega(x, y)=-\left[x+T \ln \left[1+e^{-(x-y) / T}\right]\right. \\
\left.+T \ln \left[1+e^{-(x+y) / T}\right]\right],
\end{array}
$$

with

$$
\begin{aligned}
x_{1,2} & =E, \quad x_{3,4,5}=E_{s}, \\
x_{6,7} & =\sqrt{\left[E+\frac{\left(\mu_{u r} \pm \mu_{d g}\right)}{2}\right]^{2}+\Delta^{2}}, \\
x_{8,9} & =\sqrt{\left[E+\frac{\left(\mu_{u g} \pm \mu_{d r}\right)}{2}\right]^{2}+\Delta^{2},} \\
y_{1} & =\mu_{u b}, \quad y_{2}=\mu_{d b}, \quad y_{3}=\mu_{s r}, \\
y_{4} & =\mu_{s g}, \quad y_{5}=\mu_{s b}, \quad \\
y_{6,7} & =\frac{\left(\mu_{u r}-\mu_{d g}\right)}{2}, \quad y_{8,9}=\frac{\mu_{u g}-\mu_{d r}}{2} .
\end{aligned}
$$

Here, $E=\sqrt{k^{2}+M^{2}}$ and $E_{s}=\sqrt{k^{2}+M_{s}^{2}}$, where $M_{f}=$ $m_{f}+\sigma_{f}$. Note that in the isospin limit we are working $\sigma_{u}=\sigma_{d}=\sigma$ and, thus, $M_{u}=M_{d}=M$.

The total thermodynamic potential of the quark matter phase (super-index Q) is obtained by adding to $\Omega_{q}^{M F A}$ the contribution of the leptons and a vacuum constant. Namely,

$$
\Omega^{Q}=\Omega_{q}^{M F A}+\Omega_{e}+\Omega_{\nu_{e}}-\Omega_{\mathrm{vac}}
$$

where $\Omega_{e}$ and $\Omega_{\nu_{e}}$ are the thermodynamic potentials of the electrons and neutrinos respectively. For them we use the expression corresponding to a free gas of ultrarelativistic fermions

$$
\frac{\Omega_{l}\left(T, \mu_{l}\right)}{V}=-P_{l}=-\gamma_{l}\left(\frac{\mu_{l}^{4}}{24 \pi^{2}}+\frac{\mu_{l}^{2} T^{2}}{12}+\frac{7 \pi^{2} T^{4}}{360}\right),
$$

where $P$ stands for the pressure, $l=e, \nu_{e}$, and the degeneracy factor is $\gamma_{e}=2$ for electrons and $\gamma_{\nu_{e}}=1$ for neutrinos. Notice that in Eq. (9) we have subtracted the constant $\Omega_{\mathrm{vac}} \equiv-P_{\mathrm{vac}} V$ in order to have a vanishing pressure at vanishing temperature and chemical potentials. More details are shown below in Sec. IIIC,

\section{B. Finite size effects: inclusion of MRE}

In the present work we consider the formation of finite size droplets of quark matter. The effect of finite size is included in the thermodynamic potential adopting the formalism of multiple reflection expansion (MRE; see Refs. [6 8] and references therein).

In the MRE framework, the modified density of states of a finite spherical droplet is given by

$$
\rho_{M R E}\left(k, m_{f}, R\right)=1+\frac{6 \pi^{2}}{k R} f_{S}\left(\frac{k}{m_{f}}\right)+\frac{12 \pi^{2}}{(k R)^{2}} f_{C}\left(\frac{k}{m_{f}}\right)
$$

where

$$
f_{S}\left(\frac{k}{m_{f}}\right)=-\frac{1}{8 \pi}\left(1-\frac{2}{\pi} \arctan \frac{k}{m_{f}}\right)
$$

and

$$
f_{C}\left(\frac{k}{m_{f}}\right)=\frac{1}{12 \pi^{2}}\left[1-\frac{3 k}{2 m_{f}}\left(\frac{\pi}{2}-\arctan \frac{k}{m_{f}}\right)\right]
$$

are the surface and curvature contributions to the new density of states respectively. For $f_{C}$ we employ the Madsen ansatz [6] because its functional form for any finite quark mass has not yet been derived in the MRE frame.

As shown in [8], the density of states of MRE for massive quarks is reduced compared with the bulk one, and for a range of small momentum becomes negative. The way of excluding this non physical negative density of states is to introduce an infrared cutoff in momentum space (see [8] for details). Thus, in our quark model, which includes the MRE formalism for finite spherical droplets of color superconducting quark matter, we have to perform the following replacement

$$
\int_{0}^{\Lambda} \frac{k^{2} d k}{2 \pi^{2}} \rightarrow \int_{\Lambda_{I R}}^{\Lambda} \frac{k^{2} d k}{2 \pi^{2}} \rho_{M R E} .
$$

In order to obtain the value of $\Lambda_{I R}$, we have to solve the equation $\rho_{M R E}=0$ with respect to the momentum $k$ and take the larger root as the IR cut-off. Note that $\rho_{M R E}$ depends on the quark mass and the radius of the droplets and consequently the $\Lambda_{I R}$ has the same dependence (more details are given below in Sec. IIIC).

Therefore, the full thermodynamic potential reads:

$$
\begin{aligned}
\frac{\Omega_{M R E}^{Q}}{V}= & 2 \int_{\Lambda_{I R}}^{\Lambda} \frac{k^{2} d k}{2 \pi^{2}} \rho_{M R E} \sum_{i=1}^{9} \omega\left(x_{i}, y_{i}\right) \\
& +\frac{1}{4 G}\left(\sigma_{u}^{2}+\sigma_{d}^{2}+\sigma_{s}^{2}\right)+\frac{|\Delta|^{2}}{2 H} \\
& -P_{e}-P_{\nu_{e}}+P_{\mathrm{vac}} .
\end{aligned}
$$

Multiplying on both sides of the last equation by the volume of the quark matter drop and rearranging terms we arrive to the following form for $\Omega_{M R E}^{Q}$

$$
\Omega_{M R E}^{Q}=-P^{Q} V+\alpha S+\gamma C,
$$

where the pressure $P^{Q}$ is given by

$$
\begin{aligned}
P^{Q} \equiv-\left.\frac{\partial \Omega_{M R E}^{Q}}{\partial V}\right|_{T, \mu, S, C}= & -2 \int_{\Lambda_{I R}}^{\Lambda} \frac{k^{2} d k}{2 \pi^{2}} \sum_{i=1}^{9} \omega\left(x_{i}, y_{i}\right) \\
& -\frac{1}{4 G}\left(\sigma_{u}^{2}+\sigma_{d}^{2}+\sigma_{s}^{2}\right)-\frac{|\Delta|^{2}}{2 H} \\
& +P_{e}+P_{\nu_{e}}-P_{\mathrm{vac}}, \quad(17)
\end{aligned}
$$

the surface tension is

$$
\left.\alpha \equiv \frac{\partial \Omega_{M R E}^{Q}}{\partial S}\right|_{T, \mu, V, C}=2 \int_{\Lambda_{I R}}^{\Lambda} k d k f_{S} \sum_{i=1}^{9} \omega\left(x_{i}, y_{i}\right),
$$




\begin{tabular}{c|ccccc}
\hline \hline & $m_{u, d}[\mathrm{MeV}]$ & $m_{s}[\mathrm{MeV}]$ & $\Lambda[\mathrm{MeV}]$ & $G \Lambda^{2}$ & $H / G$ \\
\hline Set 1 & 5.5 & 112.0 & 602.3 & 4.638 & $3 / 4$ \\
Set 2 & 5.5 & 110.05 & 631.4 & 4.370 & $3 / 4$ \\
\hline
\end{tabular}

TABLE I: The two sets of NJL parameters.

and the curvature energy density is

$$
\left.\gamma \equiv \frac{\partial \Omega_{M R E}^{Q}}{\partial C}\right|_{T, \mu, V, S}=2 \int_{\Lambda_{I R}}^{\Lambda} d k f_{C} \sum_{i=1}^{9} \omega\left(x_{i}, y_{i}\right) .
$$

Here we are considering a spherical drop, i.e. the area is $S=4 \pi R^{2}$ and the curvature is $C=8 \pi R$.

From the grand thermodynamic potential $\Omega_{M R E}^{Q}$ we can readily obtain the number density of quarks of each flavor and color $n_{f c} \equiv-V^{-1} \partial \Omega_{M R E}^{Q} / \partial \mu_{f c}$, the number density of electrons $n_{e}=-V^{-1} \partial \Omega_{M R E}^{Q} / \partial \mu_{e}$, and the number density of electron neutrinos $n_{\nu_{e}}=$ $-V^{-1} \partial \Omega_{M R E}^{Q} / \partial \mu_{\nu_{e}}$. The corresponding number densities of each flavor, $n_{f}$, and of each color, $n_{c}$, in the quark phase are given by $n_{f}=\sum_{c} n_{f c}$ and $n_{c}=\sum_{f} n_{f c}$ respectively. The baryon number density reads $n_{B}=$ $\frac{1}{3} \sum_{f c} n_{f c}=\left(n_{u}+n_{d}+n_{s}\right) / 3$. Finally, the Gibbs free energy per baryon is

$$
g=\frac{1}{n_{B}}\left(\sum_{f c} \mu_{f c} n_{f c}+\mu_{e} n_{e}+\mu_{\nu_{e}} n_{\nu_{e}}\right) .
$$

\section{Parametrizations}

For the NJL model, the values of the quark masses and the coupling constant $G$ can be obtained from the meson properties in the vacuum. Here we use a set of parameters taken from [18], but without the 't Hooft flavor mixing interaction. The procedure, obtained from [19], is to keep $\Lambda$ and $m$ fixed and then tune the remaining parameters $G$ and $m_{s}$ in order to reproduce $M=367.6 \mathrm{MeV}$ and $M_{s}=$ $549.5 \mathrm{MeV}$ at zero temperature and density. An estimate of $H / G$ can be obtained from Fierz transformation of the one-gluon-exchange interactions in which case one gets $H / G=0.75$. The resulting parameter sets are given in Table \. For this set of parameters we get $\Omega_{\mathrm{vac}} / V=$ $-P_{\mathrm{vac}}=-4301 \mathrm{MeV} / \mathrm{fm}^{3}$ and $-5099 \mathrm{MeV} / \mathrm{fm}^{3}$ (for set 1 and 2 respectively).

As we previously mentioned, the value of $\Lambda_{I R}$ is the largest root when solving $\rho_{M R E}=0$ with respect to $k$, depending on $m_{f}$ and $\mathrm{R}$. For a given mass, we find numerically that $\Lambda_{I R}(R)$ can be fitted as follows

$$
\Lambda_{I R}=a R^{b}
$$

with $R$ in fm and $\Lambda_{I R}$ in $\mathrm{MeV}$. The coefficients $a$ and $b$ are found to be 135.45 and -0.85 for $m_{u}=5.5 \mathrm{MeV}$; 227.72 and -0.86 for $m_{s}=110.5 \mathrm{MeV} ; 228.60$ and -0.87 for $m_{s}=112 \mathrm{MeV}$.

\section{Flavor conservation, color neutrality and other conditions}

In order to derive a quark matter EOS from the above formulae it is necessary to impose a suitable number of conditions on the variables $\left\{\mu_{f c}\right\}, \mu_{e}, \mu_{\nu_{e}}, \sigma, \sigma_{s}$ and $\Delta$. Three of these conditions are consequences from the fact that the thermodynamically consistent solutions correspond to the stationary points of $\Omega_{M R E}^{Q}$ with respect to $\sigma, \sigma_{s}$, and $\Delta$. Thus, we have

$$
\frac{\partial \Omega_{M R E}^{Q}}{\partial \sigma}=0, \quad \frac{\partial \Omega_{M R E}^{Q}}{\partial \sigma_{s}}=0, \quad \frac{\partial \Omega_{M R E}^{Q}}{\partial|\Delta|}=0 .
$$

To obtain the remaining conditions one must specify the physical situation in which one is interested in. As in previous works [1, 6, 20, 21], we are dealing here with just deconfined quark matter that is temporarily out of chemical equilibrium under weak interactions. The appropriate condition in this case is flavor conservation between hadronic and deconfined quark matter. This can be written as

$$
Y_{f}^{H}=Y_{f}^{Q} \quad f=u, d, s, e, \nu_{e}
$$

being $Y_{f}^{H} \equiv n_{f}^{H} / n_{B}^{H}$ and $Y_{i}^{Q} \equiv n_{f}^{Q} / n_{B}^{Q}$ the abundances of each particle in the hadron and quark phase respectively. It means that the just deconfined quark phase must have the same "flavor" composition than the $\beta$ stable hadronic phase from which it has been originated. Notice that, since the hadronic phase is assumed to be electrically neutral, flavor conservation ensures automatically the charge neutrality of the just deconfined quark phase. The conditions given in Eq. (23) can be combined to obtain

$$
\begin{aligned}
n_{d} & =\xi n_{u}, \\
n_{s} & =\eta n_{u}, \\
n_{\nu_{e}} & =\kappa n_{u} \\
3 n_{e} & =2 n_{u}-n_{d}-n_{s},
\end{aligned}
$$

where $n_{i}$ is the particle number density of the $i$-species in the quark phase. The quantities $\xi \equiv Y_{d}^{H} / Y_{u}^{H}, \eta \equiv$ $Y_{s}^{H} / Y_{u}^{H}$ and $\kappa \equiv Y_{\nu_{e}}^{H} / Y_{u}^{H}$ are functions of the pressure and temperature, and they characterize the composition of the hadronic phase. These expressions are valid for any hadronic EOS. For hadronic matter containing $n, p$, $\Lambda, \Sigma^{+}, \Sigma^{0}, \Sigma^{-}, \Xi^{-}$and $\Xi^{0}$, we have

$$
\begin{aligned}
\xi & =\frac{n_{p}+2 n_{n}+n_{\Lambda}+n_{\Sigma^{0}}+2 n_{\Sigma^{-}}+n_{\Xi^{-}}}{2 n_{p}+n_{n}+n_{\Lambda}+2 n_{\Sigma^{+}}+n_{\Sigma^{0}}+n_{\Xi^{0}}}, \\
\eta & =\frac{n_{\Lambda}+n_{\Sigma^{+}}+n_{\Sigma^{0}}+n_{\Sigma^{-}}+2 n_{\Xi^{0}}+2 n_{\Xi^{-}}}{2 n_{p}+n_{n}+n_{\Lambda}+2 n_{\Sigma^{+}}+n_{\Sigma^{0}}+n_{\Xi^{0}}}, \\
\kappa & =\frac{n_{\nu_{e}}^{H}}{2 n_{p}+n_{n}+n_{\Lambda}+2 n_{\Sigma^{+}}+n_{\Sigma^{0}}+n_{\Xi^{0}}} .
\end{aligned}
$$

Additionally, the deconfined phase must be locally colorless; thus it must be composed by an equal number of 
red, green and blue quarks

$$
n_{r}=n_{g}=n_{b} .
$$

Also, $u r, u g, d r$, and $d g$ pairing will happen provided that $|\Delta|$ is nonzero, leading to

$$
n_{u r}=n_{d g}, \quad n_{u g}=n_{d r} .
$$

In order to have all Fermi levels at the same value, we consider [5]

$$
n_{u g}=n_{u r}, \quad n_{s b}=n_{s r}
$$

These two equations, together with Eqs. (31) and (32) imply that $n_{u r}=n_{u g}=n_{d r}=n_{d g}$ and $n_{s r}=n_{s g}=n_{s b}$ [5].

Finally, including the conditions Eqs. (22) we have 13 equations involving the 14 unknowns $\left(\sigma, \sigma_{s},|\Delta|, \mu_{e}, \mu_{\nu_{e}}\right.$ and $\left.\left\{\mu_{f c}\right\}\right)$. For given value of one of the chemical potentials $\left(\right.$ e.g. $\left.\mu_{u r}\right)$, the set of equations can be solved once the values of the parameters $\xi, \eta, \kappa$, the temperature $T$ and the radius of the drop $R$ are given. Instead of $\mu_{u r}$, we can provide a value of the Gibbs free energy per baryon $g_{\text {quark }}$ and solve simultaneously Eqs. (24)-(33) together with Eqs. (22) in order to obtain $\sigma, \sigma_{s},|\Delta|, \mu_{e}, \mu_{\nu_{e}}$ and $\left\{\mu_{f c}\right\}$.

\section{DECONFINEMENT OF COLOR SUPERCONDUCTING DROPLETS IN PROTO-NEUTRON STAR MATTER}

The total thermodynamic potential of a quark matter drop immersed in an homogeneous hadronic environment is $\Omega=\Omega^{H}+\Omega_{M R E}^{Q}$, where the indexes $H$ and $Q$ refer to the hadronic and the quark phase respectively. For the hadronic phase we have $\Omega^{H}=-P^{H} V^{H}$ and for the quark phase we have $\Omega_{M R E}^{Q}=-P^{Q} V^{Q}+\alpha S+\gamma C$.

The condition of mechanical equilibrium is given by [6, 22]:

$$
\left.\frac{\partial \Omega}{\partial R}\right|_{T, \mu, V}=\left.\frac{\partial \Omega^{H}}{\partial R}\right|_{T, \mu, V}+\left.\frac{\partial \Omega_{M R E}^{Q}}{\partial R}\right|_{T, \mu, V}=0 .
$$

Since $V=V^{Q}+V^{H}$ is constant, we have:

$$
-P^{Q} \frac{d V^{Q}}{d R}+\alpha \frac{d S}{d R}+\gamma \frac{d C}{d R}+P^{H} \frac{d V^{Q}}{d R}=0 .
$$

Thus, for a spherical droplet the condition for mechanical equilibrium reads (c.f. [] $]$ ):

$$
P^{Q}-\frac{2 \alpha}{R}-\frac{2 \gamma}{R^{2}}-P^{H}=0 .
$$

Notice that in the bulk limit $R \rightarrow \infty$ we find the standard condition $P^{H}=P^{Q}$.

Additionally, we assume thermal and chemical equilibrium, i.e. the Gibbs free energy per baryon are the same for both hadronic matter and quark matter at a given common temperature. Thus, we have

$$
g^{H}=g^{Q}, \quad T^{H}=T^{Q} .
$$

If we fix the radius $R$ of the deconfined drop for a given temperature $T^{H}$ and neutrino chemical potential of the trapped neutrinos in the hadronic phase $\mu_{\nu_{e}}^{H}$, there is an unique hadronic pressure $P^{H}$ at which the equilibrium conditions are fulfilled. Notice that, differently than in the bulk case studied in [1], $P^{H}$ and $P^{Q}$ are not equal because of the appearance of a surface and a curvature term in the condition for mechanical equilibrium. Also, the mass-energy density $\rho^{H}$ and $\rho^{Q}$ at the equilibrium point are different in general. Similarly, while the abundance $Y_{\nu_{e}}$ of neutrinos is the same in both the hadronic and just deconfined quark phases, the chemical potentials $\mu_{\nu_{e}}^{Q}$ and $\mu_{\nu_{e}}^{H}$ are different.

In the present work we want to describe thermodynamic conditions analogous to those encountered in protoneutron stars. From numerical simulations of the first tens of seconds of evolution [23], we know that the protoneutron star cools from $T \sim 40 \mathrm{MeV}$ to temperatures below $2-4 \mathrm{MeV}$ in about one minute. In that period, the chemical potential $\mu_{\nu_{e}}^{H}$ of the trapped neutrinos decreases from $\sim 200 \mathrm{MeV}$ to essentially zero. Then, taking into account typical protoneutron star conditions, we have solved Eqs. (36) and (37) together with the conditions showed in Sections II and III, for temperatures in the range $0-60 \mathrm{MeV}, \mu_{\nu_{e}}^{H}$ in the range $0-200 \mathrm{MeV}$ and for different radii of the color superconducting droplets. The results are displayed in Figs. 1 and 2 for the parameterizations of the equations of state given in previous sections.

In Fig. 1 we show the mass-energy density of hadronic matter (in units of the nuclear saturation density $\rho_{0}=$ $2.7 \times 10^{14} \mathrm{~g} \mathrm{~cm}^{3}$ ) above which it is energetically favorable the conversion of a portion of hadronic matter into an identical deconfined drop (i.e. with the same $T, P, g$, $Y_{f}$ and radius $R$ ). For the calculations we considered the following possibilities: set 1 of the NJL model in the left panels and set 2 in the right panels, temperatures in the range $0-60 \mathrm{MeV}$, and neutrino chemical potential $\mu_{\nu_{e}}^{H}=$ $0,100,200 \mathrm{MeV}$. Within each plot we consider different values of $R$; from right to left the curves correspond to $R[\mathrm{fm}]=2,5,10,20,50,500, \infty$. Notice that, in the six panels of Fig. 1, the curves for the bulk case $(R=\infty)$ are almost coincident with the curves for $R=500 \mathrm{fm}$. The main observed effect in Fig. 1 is that the transition density increases considerably for small radii. This is a consequence of the significant increase of the surface tension $\alpha$ and the curvature energy $\gamma$ for small $R$ (see Fig. 2).

In Fig. 2 we display the surface tension $\alpha$ and the curvature energy $\gamma$ as a function of temperature for different radii of the droplet and with the set of $\left\{\mu_{f c}, \sigma, \sigma_{f}, \Delta\right\}$ that arises from the equilibrium condition discussed before. Notice that $\alpha$ and $\gamma$ do not depend explicitly on the radius $R$ of the drop (see Eqs. (18) and (19)), but for a 

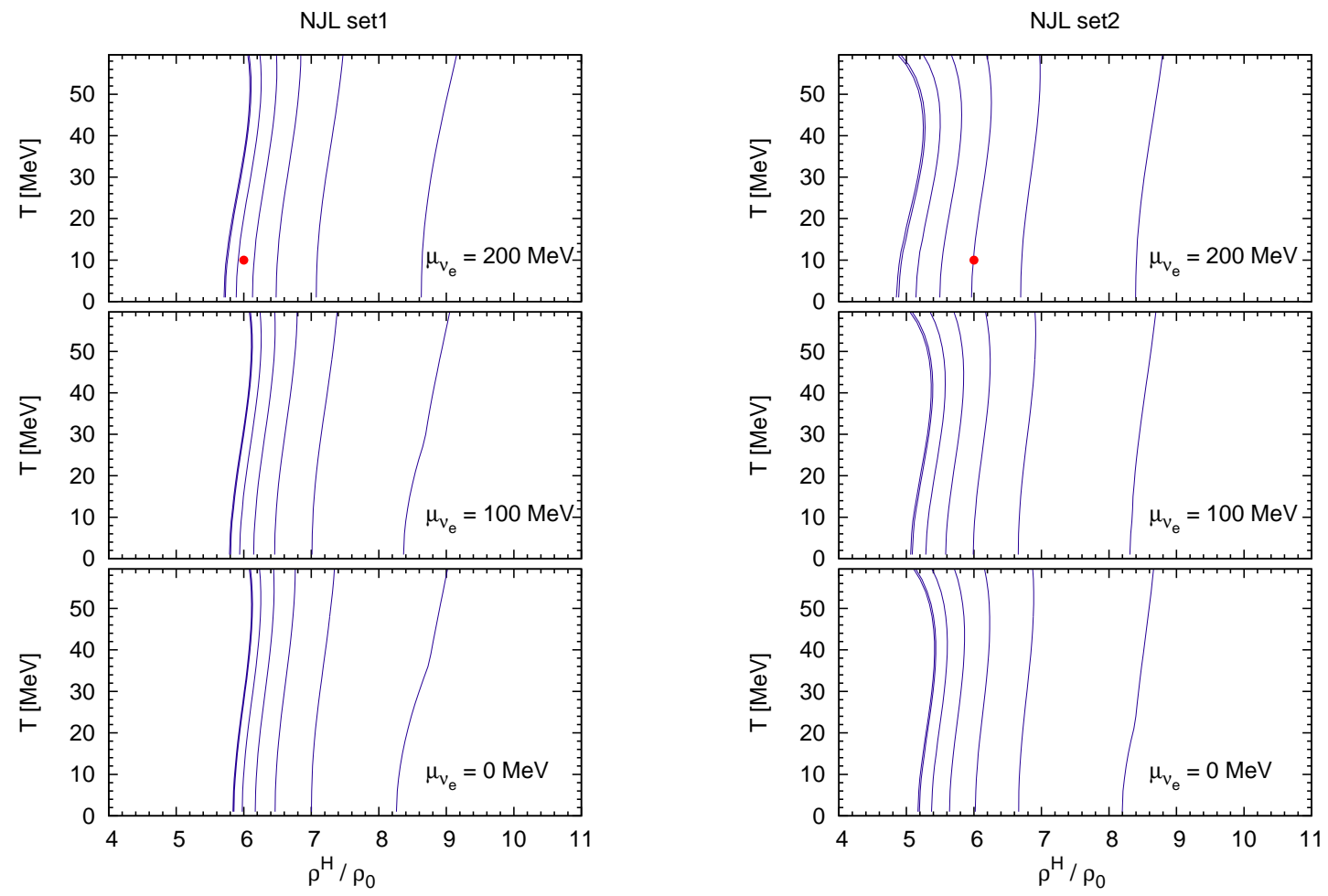

FIG. 1: For different temperatures we show the mass-energy density $\rho^{H}$ of hadronic matter above which it is energetically favorable the conversion of the hadronic phase into deconfined drops of radius $R . \rho_{0}=2.7 \times 10^{14} \mathrm{~g} \mathrm{~cm}^{3}$ is the nuclear saturation density. For the hadronic phase we employ the model introduced in Sec. II. For the quark phase we use set 1 of the NJL model presented in Sec III in the left panels, and set 2 in the right panels. In each figure the neutrino chemical potential in the hadronic phase is fixed to the following values: $\mu_{\nu_{e}}^{H}=0,100,200 \mathrm{MeV}$. Within each plot, each curve correspond to a different value of $R$. From right to left we have $R[\mathrm{fm}]=2,5,10,20,50,500, \infty$. Notice that in all figures the curves for the bulk case $(R=\infty)$ are almost coincident with the curves for $R=500 \mathrm{fm}$. A possible interpretation of these figures is best illustrated with an example: let us consider a hadronic system with a density $\rho^{H} \approx 6 \times \rho_{0}$ at a temperature $T=10 \mathrm{MeV}$ and with trapped neutrinos having a chemical potential $\mu_{\nu_{e}}^{H}=200 \mathrm{MeV}$. This state is represented by a circle in the two upper panels of this figure. Looking at the position of the circle with respect to the curves corresponding to different radii, we see that it is energetically favorable to convert hadronic matter into quark drops provided they have a radii $R \gtrsim 50 \mathrm{fm}$ (if quark matter is described by the NJL set $1 \mathrm{EoS}$ ) and $R \gtrsim 10 \mathrm{fm}$ (using NJL set $2 \mathrm{EoS}$ ). That is, radii whose curves lie to the left of the circle are energetically favored while those on the right are forbidden because of the energy cost of forming a surface. A more realistic interpretation of the figure is given in Sec. V.

given $R, T$ and $\mu_{\nu_{e}}^{H}$ there is a unique set of $\left\{\mu_{f c}, \sigma, \sigma_{f}, \Delta\right\}$ satisfying the equilibrium conditions, i.e. $\alpha$ and $\gamma$ depend on $R$ through $\left\{\mu_{f c}, \sigma, \sigma_{f}, \Delta\right\}$. From Fig. 2 we see that while both $\alpha$ and $\gamma$ are roughly independent of the temperature, there is a larger dependence with the radius $R$ leading to the behavior of the transition density explained in the caption of Fig. 1. Notice also that the here-found values of $\alpha$ are larger that those found within the MIT Bag model which are typically $\sim 30-60 \mathrm{MeV}$ $\mathrm{fm}^{-2}$ for drops larger than few fm [24].

\section{FLUCTUATIONS AND DECONFINEMENT}

According to the theory of homogeneous nucleation, the free energy involved in the formation of a spherical quark bubble of radius $R$ is given by [25]

$$
\Delta \Omega=-\frac{4 \pi}{3} R^{3} \Delta P+4 \pi \alpha R^{2}+8 \pi \gamma R,
$$

where $\Delta P=P^{Q}-P^{H}$ is the pressure difference between internal and external parts of the bubble. For given $\Delta P$, $\alpha$ and $\gamma$, the extremal points (maximum or minimum) of $\Delta \Omega$ are obtained from $\partial \Delta \Omega / \partial R=0$, which leads to Eq. 

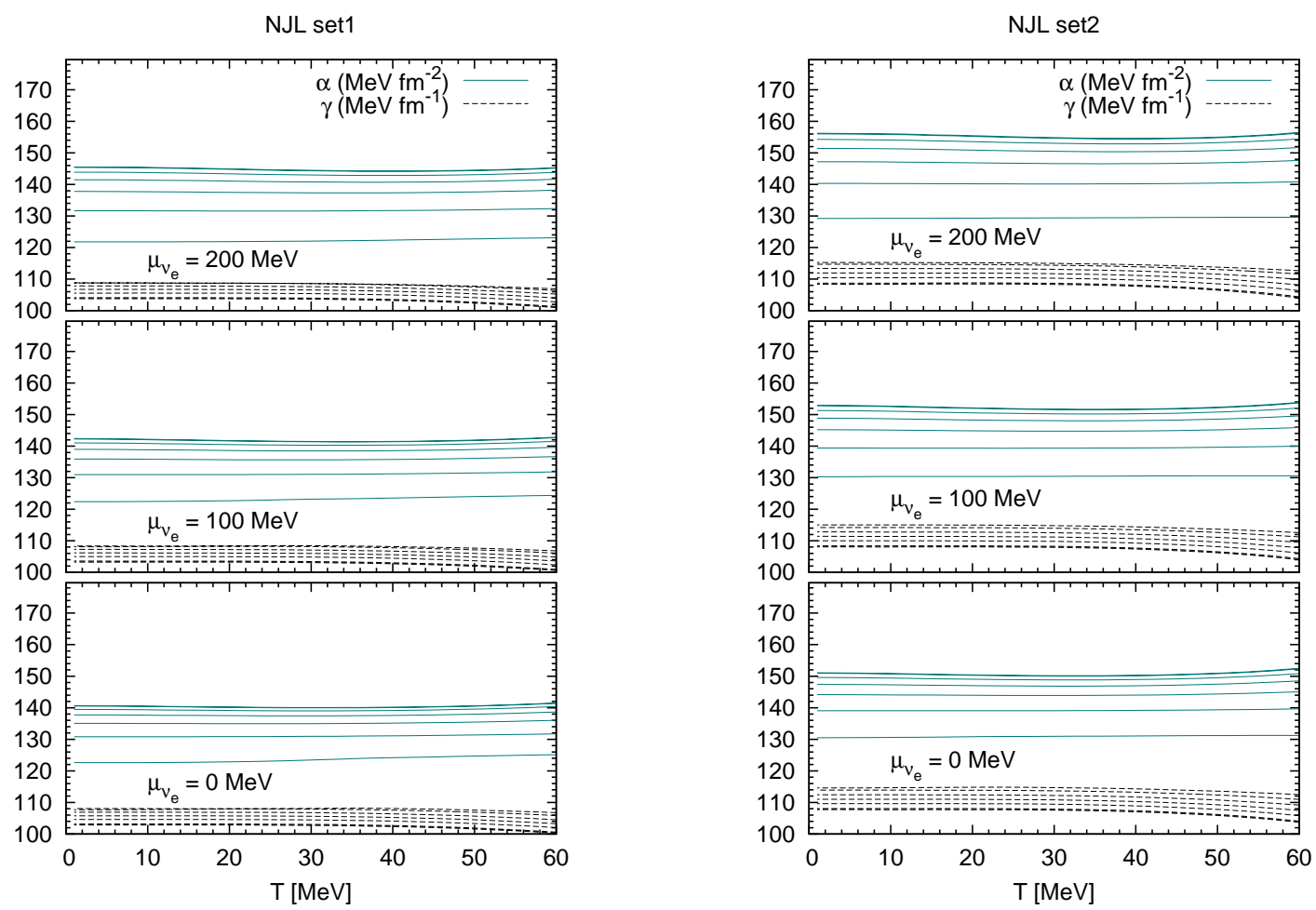

FIG. 2: The surface tension $\alpha$ and the curvature energy $\gamma$ as a function of temperature for different radii of the droplet and with the set of $\left\{\mu_{f c}, \sigma, \sigma_{f}, \Delta\right\}$ that arises from the equilibrium conditions leading to Fig. 1. Different curves correspond to different radii $R$; in the case of $\alpha$ we have $R[\mathrm{fm}]=2,5,10,20,50,500, \infty$ from bottom to top while in the case of $\gamma$ the values are the same but from top to bottom. Both $\alpha$ and $\gamma$ are roughly independent of the temperature but there is a larger dependence with $R$.

(36). Thus, the critical radii are given by:

$$
R_{ \pm}=\frac{\alpha}{\Delta P}(1 \pm \sqrt{1+b})
$$

with $b \equiv 2 \gamma \Delta P / \alpha^{2}$.

For $b<-1$ both solutions are complex and $\Delta \Omega$ is a monotonically decreasing function of $R$. This means that any small fluctuation of one phase into the other will gain energy by expanding and a rapid phase transition is likely to occur. For $b \geq-1, \Delta \Omega$ has a local minimum at $R_{-}$and a local maximum at $R_{+}$. For $\Delta \Omega\left(R_{+}\right)<0$ we have again that any small fluctuation is energetically favored. For $\Delta \Omega\left(R_{+}\right)>0$ bubbles with radii larger than $R_{+}$gain energy by growing unlimitedly, while those below the critical size gain energy by shrinking to zero (if $R_{-}<0$ ) or to $R_{-}$(if $R_{-}>0$ ). In this case, the standard assumption in the theory of bubble nucleation in first order phase transitions is that bubbles form with a critical radius $R_{+}$[25].

The approach we adopted in the previous section is closely related to what we explained in the above paragraph. Instead of finding the critical radius for arbitrary values of $\Delta P, \alpha$ and $\gamma$, we fixed $R$ and found the corresponding $\Delta P, \alpha$ and $\gamma$ that satisfy the conditions presented in Secs. II, III and IV. Since Eq. (36) is satisfied by construction, the radius $R$ is precisely the critical radius $R_{+}$introduced in Eq. (39), given that we choose the solution that verifies $\partial^{2} \Delta \Omega / \partial R^{2}<0$.

In the light of the previous discussion we may give another interpretation to the results presented in Fig. 1. Let us consider, for simplicity, a uniform hadronic system with trapped neutrinos having a chemical potential $\mu_{\nu_{e}}^{H}$ and a constant mass-energy density infinitesimally to the right of the curve with $R=\infty$ in the $T-\rho^{H} / \rho_{0}$ plane corresponding to the same $\mu_{\nu_{e}}^{H}$. Since this density corresponds to the bulk transition we shall refer to it as $\rho_{b u l k}^{H}\left(T, \mu_{\nu_{e}}^{H}\right)$. For such a density, it is favorable to convert the system into quark matter in bulk, but this is not possible in practice due to the surface and curvature energy cost.

However, fluctuations in the independent thermodynamic variables $\left\{T, \rho^{H}, \mu_{\nu_{e}}^{H}\right\}$ of the hadronic fluid may drive some portion of it to a state described by $\{T+$ 

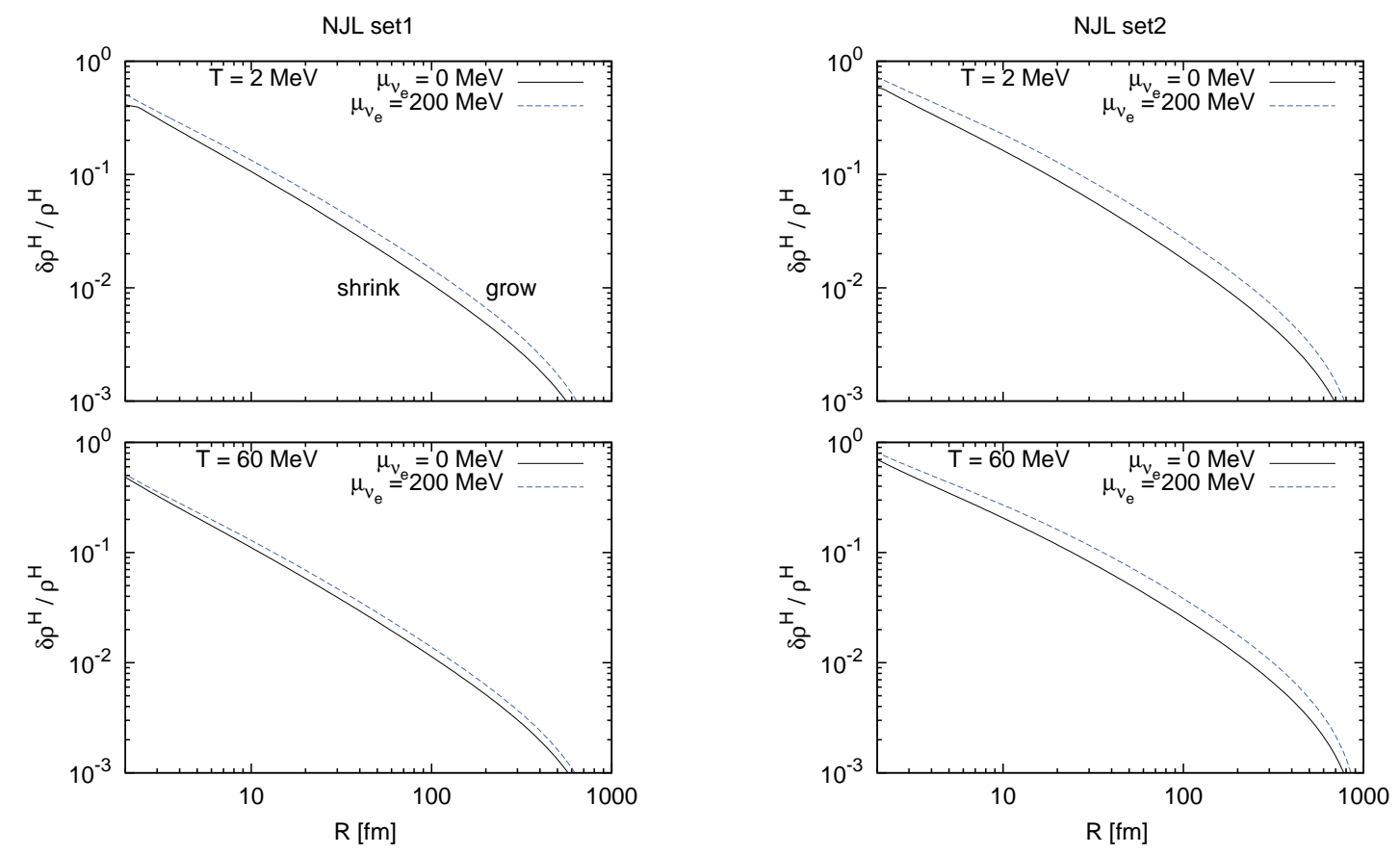

FIG. 3: Critical spectrum for fluctuations that allow deconfinement of hadronic matter in a protoneutron star. As in the previous figures, the results were calculated using GM4 + NJL set1 on the left panel and GM4 + NJL set 2 on the right panel. The spectrum does not depend significantly on the temperature and the chemical potential of trapped neutrinos. Fluctuations in hadronic matter having a given $\delta \rho^{H} / \rho^{H}$ are able to grow if they have a size $R$ larger than the here-shown critical one.

$\left.\delta T, \rho^{H}+\delta \rho^{H}, \mu_{\nu_{e}}^{H}+\delta \mu_{\nu_{e}}^{H}\right\}$. Nevertheless, notice that the curves of Fig. 1 are quite vertical, i.e. the transition is not very sensitive to changes in $T$. The same holds for variations in $\mu_{\nu_{e}}^{H}$. Thus, we shall consider only energydensity fluctuations with radius $R_{f l}$ that drive some part of the hadron fluid to a density $\rho_{*}^{H}=\rho_{b u l k}^{H}+\delta \rho^{H}$ to the right of a curve with a given $R$. As explained in the caption of Fig. 1, quark drops with radii larger than $R$ are energetically favored. Thus, if the fluctuation has a size $R_{f l}$ larger than $R$ it will be energetically favorable for it to grow indefinitely. In order to quantify this, we calculate the difference $\delta \rho^{H}$ between $\rho_{b u l k}^{H}$ and the hadronic density of the point that allows nucleation with radius $R$ or larger. In such a way we can construct a critical spectrum $\delta \rho^{H} / \rho^{H}$ as a function of $R$ for different values of $T$ and $\mu_{\nu_{e}}^{H}$ as seen in Fig. 3. Fluctuations of a given over-density $\delta \rho^{H} / \rho^{H}$ must have a size larger than the critical value given in Fig. 3 in order to grow. Equivalently, fluctuations of a given size must have an over-density $\delta \rho^{H} / \rho^{H}$ larger than the critical one for that size.

We can calculate the formation rate of critical bubbles through

$$
\Gamma \approx T^{4} \exp \left(-\delta \Omega_{c} / T\right)
$$

where in our case $\delta \Omega_{c}$ is the work required to form a quark bubble with the critical radius from hadronic matter at the bulk transition point

$$
\delta \Omega_{c} \equiv-\frac{4 \pi}{3} R^{3}\left(P^{Q}-P_{b u l k}^{H}\right)+4 \pi \alpha R^{2}+8 \pi \gamma R
$$

Instead of $T^{4}$, different prefactors are used for $\Gamma$ in other works (see e.g. [2]). However, this fact does not affect significantly the results because $\Gamma$ is largely dominated by the exponent in Eq. (40); i.e. we always have $\log _{10} \Gamma \approx$ $\log _{10}$ (prefactor) $-\delta \Omega_{c} /[T \ln (10)]$ with the second term much larger than the first.

The results are given in Fig. 4 and show that critical bubbles with $R \gtrsim 800 \mathrm{fm}$ are strongly disfavored while those with $R \lesssim 800 \mathrm{fm}$ have a huge rate. In practical situations, i.e. at neutron star cores, this means that if fluctuations lead hadronic matter to the bulk transition point, quark drops with $R \lesssim 800 \mathrm{fm}$ will nucleate instantaneously. 

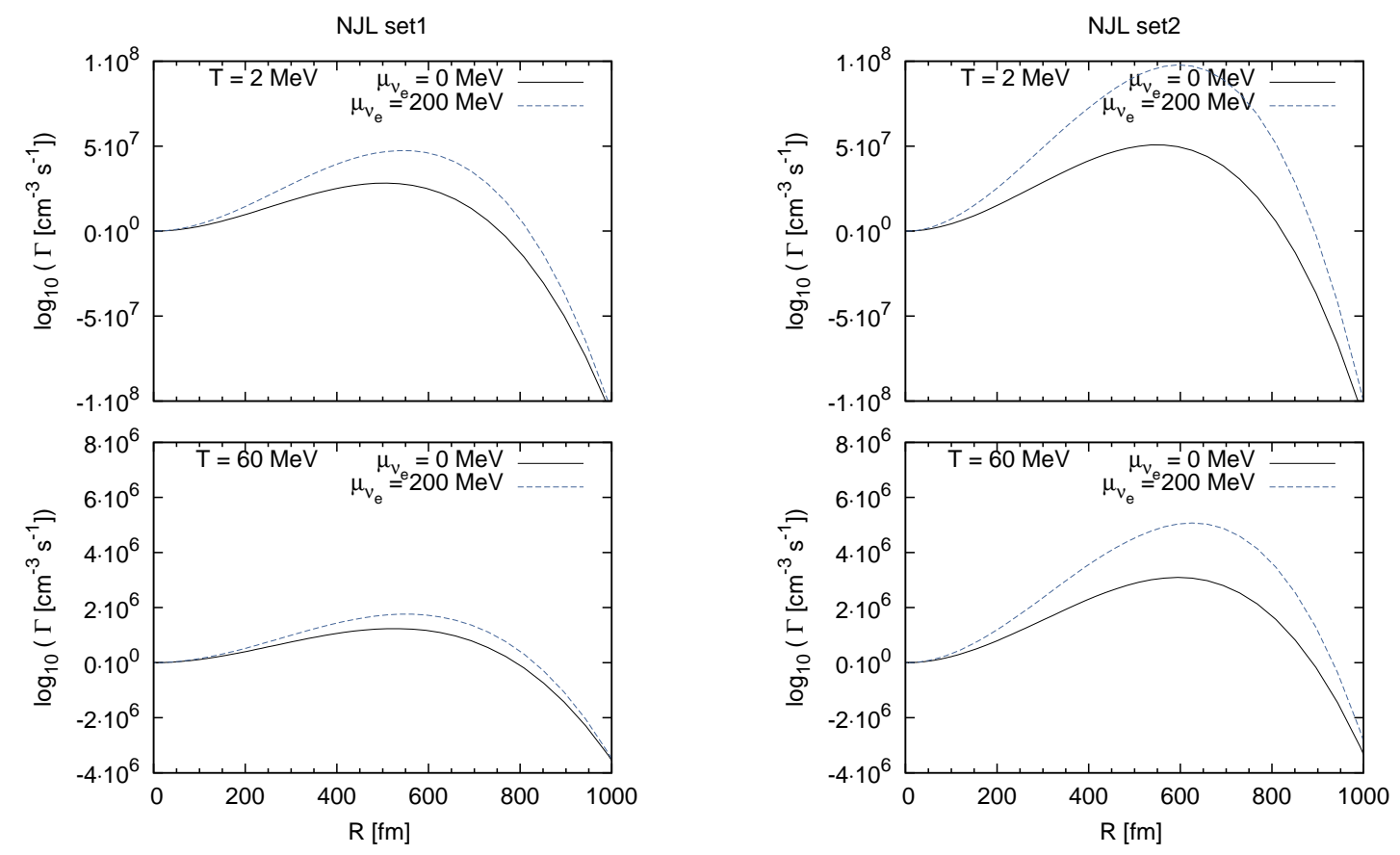

FIG. 4: Nucleation rate for bubbles of the critical size.

\section{SUMMARY AND CONCLUSIONS}

In the present paper we have studied the deconfinement of quark matter in protoneutron stars employing a two-phase description of the first order phase transition. For the hadronic phase we used the non-linear Walecka model (Sect. II) which includes the whole baryon octet, electrons and trapped electron neutrinos in equilibrium under weak interactions. For the just deconfined quark matter we used a $S U(3)_{f}$ NJL model including color superconducting quark-quark interactions. In this phase, finite size effects are included via the multiple reflection expansion framework (Sect. III). We considered that color superconducting quark droplets are formed in mechanical and thermal equilibrium with the hadronic environment when the Gibbs free energy of both phases are equal. Also, since deconfinement is a strong interaction process, we consider that the just formed quark phase has the same flavor composition than the hadronic $\beta$-stable phase, and consequently it is out of chemical equilibrium under weak interactions. The further $\beta$-equilibration of such quark phase is not addressed within the present paper.

Through this model we determined the density of hadronic matter at which deconfinement is possible for different radii of the just formed quark drops, as well as for different values of the temperature and the chemical potential of the trapped neutrinos (see Fig. 1). We have used typical conditions expected in the interior of protoneutron stars, i.e. temperatures in the range of $0-60$ $\mathrm{MeV}$ and chemical potentials of the trapped neutrino gas up to $200 \mathrm{MeV}$. As expected, we find that the transition density increases significantly when the radius of the droplet decreases. This is a consequence of the increase of the surface tension $\alpha$ and the curvature energy $\gamma$ for small radii. Additionally, we found that $\alpha$ and $\gamma$ at the deconfinement point are almost independent of the temperature and the neutrino chemical potential (see Fig. $2)$.

When the bulk transition density is reached in the core of a neutron star, it is energetically favored to convert a macroscopically large portion of hadronic matter into quark matter. But in practice, it is needed some overdensity with respect to the bulk transition density in order to compensate the surface and curvature energy cost of a finite drop. Since this energy cost depends on the drop radius, so does the necessary over-density and overpressure necessary to nucleate it. Thus, we can derive a critical fluctuation spectrum $\delta \rho^{H} / \rho^{H}$ versus $R$ delimiting which fluctuations are able to grow unlimitedly and which will shrink (see Fig. 3). Typically, fluctuations of $\delta \rho^{H} / \rho^{H} \sim 0.001-0.1$ above the bulk point are needed for 
the nucleation of drops with $R \sim 10-1000 \mathrm{fm}$. However, the nucleation rates $\Gamma$ vary over several orders of magnitude. Our results show that drops with $R \sim 2-800 \mathrm{fm}$ have a huge nucleation rate while those with $R \gtrsim 800 \mathrm{fm}$ are strongly suppressed (see Fig. 4).

We have also shown that fluctuations in the temperature and in the chemical potential of trapped neutrinos are not very important for deconfinement. This is in contrast with previous results found within the frame of the MIT Bag model (e.g. in 26] it is argued that nucleation is suppressed at $T \lesssim 2 \mathrm{MeV}$ and in [20] it is found that neutrino trapping precludes deconfinement). Instead, fluctuations in the energy density are the more efficient way to trigger the transition.

Notice that the nucleation rate and the typical radii of deconfined drops are also very different from the values found within the MIT Bag model (see e.g. 2] and references therein). The drops studied in [2] have typi- cally radii less than $10 \mathrm{fm}$ and a long nucleation time, in contrast ours may have much larger radii and nucleate almost instantaneously. This is due to the use of different equations of state for the quark phase as well as for the different treatments of the surface and curvature terms. While in [2] the surface tension is assumed to be constant $\left(\alpha=30 \mathrm{MeV} \mathrm{fm}^{-2}\right)$, in our work $\alpha$ and $\gamma$ are calculated self consistently within the MRE formalism resulting non-constant values around $140 \mathrm{MeV} \mathrm{fm}^{-2}$ and $110 \mathrm{MeV} \mathrm{fm}^{-1}$ respectively. Since our surface tension is larger, larger critical drops are obtained. The typical $\left|\delta \Omega_{c}\right|$ is also larger and results in huge nucleation rates. In the context of protoneutron stars the main conclusion is that if the bulk transition point is attained near the star centre, quark matter drops with $R \lesssim 800 \mathrm{fm}$ will nucleate instantaneously. Since the bulk transition density is $\sim 5-6 \rho_{0}$, this should happen for stars with masses larger than $\sim 1.5-1.6 M_{\odot}$.
[1] G. Lugones, T. A. S. do Carmo, A. G. Grunfeld and N.N. Scoccola, Phys. Rev. D 81, 085012 (2010)

[2] I. Bombaci, D. Logoteta, P.K. Panda, C. Providencia, I. Vidana, Phys. Lett. B 680, 448 (2009)

[3] B. W. Mintz, E. S. Fraga, G. Pagliara, J. SchaffnerBielich, Phys. Rev. D81, 123012 (2010).

[4] M. Hempel, G. Pagliara, J. Schaffner-Bielich, Phys. Rev. D80, 125014 (2009).

[5] G. Lugones and I. Bombaci, Phys. Rev. D 72, 065021 (2005).

[6] J. Madsen, Phys. Rev. D 50, 3328 (1994).

[7] O. Kiriyama and A. Hosaka, Phys. Rev. D 67, 085010 (2003).

[8] O. Kiriyama, Phys. Rev. D 72, 054009 (2005).

[9] J.D. Walecka, Ann. Phys. 83, 491 (1974); B.D. Serot and J.D. Walecka, Adv. Nucl. Phys. 16, 1 (1986).

[10] N. K. Glendenning and S.A. Moszkowski, Phys. Rev. Lett. 67, 2414 (1991).

[11] J. Boguta and A. R. Bodmer, Nucl. Phys. A 292, 413 (1977)

[12] D.P. Menezes and C. Providência, Phys. Rev. C 68, 035804 (2003); A.M.S. Santos and D.P. Menezes, Phys. Rev. C 69, 045803 (2004).

[13] P. Demorest, Nature 467, 1081-1083, (2010).

[14] M. Huang, P. F. Zhuang and W. F. Chao, Phys. Rev. D 67, 065015 (2003).

[15] S. B. Ruester, V. Werth, M. Buballa, I. A. Shovkovy and D. H. Rischke, Phys. Rev. D 72, 034004 (2005).

[16] D. Blaschke, S. Fredriksson, H. Grigorian, A. M. Oztas and F. Sandin, Phys. Rev. D 72, 065020 (2005).

[17] M. Ciminale, R. Gatto, N. D. Ippolito, G. Nardulli, M. Ruggieri, Phys. Rev. D77, 054023 (2008).

[18] T. Hatsuda and T. Kunihiro, Phys. Rept. 247, 221 (1994).

[19] M. Buballa, Phys. Rept. 407, 205 (2005).

[20] G. Lugones and O. G. Benvenuto, Phys. Rev. D 58, 083001 (1998); O. G. Benvenuto and G. Lugones, Mon. Not. R.A.S. 304, L25 (1999);

[21] K. Iida and K. Sato, Phys. Rev. C 58, 2538 (1998); I. Bombaci, I. Parenti, I. Vidaña, Astrophys.J. 614, 314 (2004); I. Bombaci, G. Lugones, I. Vidaña, Astronomy and Astrophysics 462, 1017 (2007); G. Lugones, A. G. Grunfeld, N.N. Scoccola and C. Villavicencio, Phys. Rev. D 80, 045017 (2009)

[22] L.D. Landau, E.M. Lifshitz (1980). Statistical Physics, Part 1. Vol. 5 (3rd ed.). Butterworth-Heinemann. ISBN 978-0-750-63372-7.

[23] A. Burrows and J. M. Lattimer, Astrophys. J. 307, 178 (1986); W. Keil and H-Th. Janka, Astron. Astrophys., 296, 145 (1995); J. A. Pons et al., Astrophys. J. 513, 780, (1999).

[24] X. J. Wen, J. Y. Li, J. Q. Liang, and G. X. Peng, Phys. Rev. C 82, 025809 (2010)

[25] J. Olesen and J. Madsen, Phys. Rev. D 49, 2698 (1994).

[26] J. E. Horvath, O. G. Benvenuto and H. Vucetich, Phys. Rev. D 45, 3865 (1992). 